\title{
Luminescence of Atomic Barium in Rare Gas Matrices: A Two- Dimensional Excitation/Emission Spectroscopy Study
}

\author{
Barry Davis and John G. McCaffrey*(1) \\ Department of Chemistry, Maynooth University, National University of Ireland, Maynooth, County Kildare, Ireland
}

\begin{abstract}
A detailed characterization is made of the distinct sites occupied by atomic barium isolated in the three rare gas hosts $\mathrm{Ar}, \mathrm{Kr}$, and Xe in excitation scans extracted from the recorded total $6 s 6 \mathrm{p}^{1} \mathrm{P}_{1}$ $\rightarrow(6 s)^{2}{ }^{1} \mathrm{~S}_{0}$ fluorescence. Extensive use has been made of twodimensional excitation/emission (2D-EE) spectroscopy to achieve a comprehensive characterization for the wide variety of sites present in the $\mathrm{Ba} / \mathrm{RG}$ matrix systems. The $2 \mathrm{D}-\mathrm{EE}$ technique has proved to be a very powerful method to probe the effects of strong intersite reabsorption when extensive spectral overlap occurs between emission and resonance $6 \mathrm{~s} 6 \mathrm{p}{ }^{1} \mathrm{P}_{1} \leftarrow(6 \mathrm{~s})^{2}{ }^{1} \mathrm{~S}_{0}$ absorption of barium atoms occupying multiple sites. Two-dimensional excitation/emission scans have also been used in this study to monitor the effects of sample

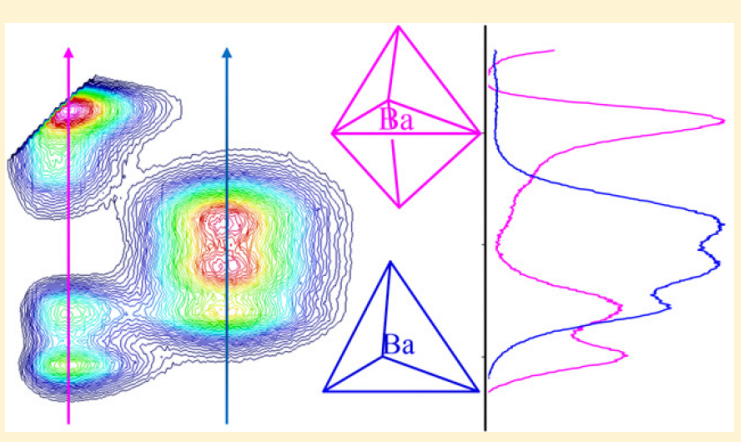
annealing and thereby identify the thermally stable sites of isolation. Sites of the same type occupied by atomic barium in the three host solids are identified in resolved excitation spectra and are associated on the basis of the observed matrix shift versus host polarizability. Following site associations, the photophysical properties of each matrix site were characterized revealing that the Stokes shift was greatest in the blue site, smallest for the violet site, and intermediate for the green site. The emission temperature dependences and excited state lifetimes were recorded, indicating that measured radiative lifetimes of 4-5ns were in good agreement with the gas phase value of $8.4 \mathrm{~ns}$ when corrected for the effective field of the solids. The only exception to this was the blue site in $\mathrm{Ba} / \mathrm{Xe}$, where a nonradiative quenching channel exists even at $9.8 \mathrm{~K}$ that competes effectively with the nanosecond fluorescence. An unusual, asymmetric $2+1$ excitation band has been recorded for atomic barium in the three rare gas hosts in addition to the threefold split, Jahn-Teller bands typically observed for $\mathrm{P} \leftarrow \mathrm{S}$ absorptions of matrix-isolated metal atoms. Possible assignments of the sites responsible for these band shapes are made on the basis of recent spectral simulations obtained from molecular dynamics calculations on the $\mathrm{Ba} / \mathrm{Xe}$ system.
\end{abstract}

\section{INTRODUCTION}

This article presents an overview of the total luminescence recorded for barium atoms isolated in solid $\mathrm{Ar}, \mathrm{Kr}$, and $\mathrm{Xe}$ and a detailed examination of the distinct excitation bands arising from the variety of sites occupied by this guest metal atom in the three solid rare gases. With the inclusion of data for the $\mathrm{Ba} / \mathrm{Ar}$ and $\mathrm{Ba} / \mathrm{Kr}$ systems, the present contribution expands the matrix emission data and, in so doing, provides a more comprehensive analysis of the site attributions published by us recently for $\mathrm{Ba} / \mathrm{Xe}{ }^{1}$ The first $\mathrm{Ba} /$ rare gas (RG) matrix luminescence spectra were reported more than three decades ago by Balling and $\mathrm{Wright}^{2}(\mathrm{~B} \& \mathrm{~W})$, while results for $\mathrm{Ba} / \mathrm{Ar}$ and $\mathrm{Ba} / \mathrm{Xe}$ were published recently by Fairbank and coworkers. ${ }^{3} \mathrm{~B} \& W$ 's emission spectra are, however, not consistent with those recorded in the present study as shown by the red traces in Figure 1, even though both were produced with photoexcitation of the dominant (6s6p) ${ }^{1} \mathrm{P}_{1}$ absorption bands. The most significant difference is that our $\mathrm{Ba} / \mathrm{Ar}$ spectrum shows a pair of resolved emission bands located at 537 and 550 while that published in 1985 by Balling and Wright ${ }^{2}$ consisted of a single, longer wavelength band at approximately $568 \mathrm{~nm}$. The reason for the pronounced difference is, as will be shown in this work, the matrix samples prepared in B\&W's study were strongly absorbing which had the effect that complete reabsorption of the blue emission features occurred leaving only emission from the band furthest to the red. The authors $\mathrm{B} \& W$ acknowledged that the observed emission probably originated from a single site, with the intensity of this band enhanced by the successive reabsorptions of higher energy emission bands. In an effort to identify all of the site-related $\mathrm{Ba} / \mathrm{RG}$ emission bands-especially the emission features furthest to the blue-samples having low optical densities $(\mathrm{OD}<0.5)$ were prepared and the total ${ }^{1} \mathrm{P}_{1}$ state luminescence was recorded with two-dimensional excitation-emission (2DEE) spectroscopy. Fortunately, the deposition conditions required to achieve a high degree of atomic isolation result in relatively weakly absorbing samples. In addition, strong matrix annealing was used to further simplify the emission spectra by removing thermally unstable sites, thereby reducing the number of features present in the complex absorption spectra.

Based on absorption data, it was proposed by us in an earlier paper $^{4}$ that three thermally stable sites of isolation exist for Ba in solid $\mathrm{Ar}$ and $\mathrm{Kr}$ and at least two sites occur in Xe. This

Received: May 29, 2018

Revised: August 23, 2018

Published: August 24, 2018 


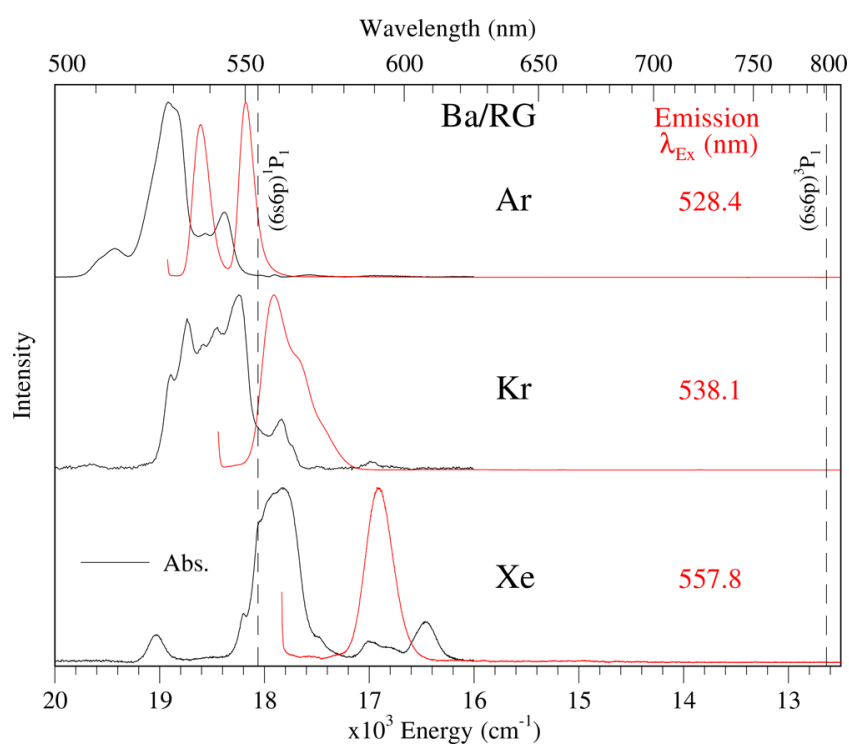

Figure 1. Absorption and emission spectra recorded at $10 \mathrm{~K}$ for annealed $\mathrm{Ba} / \mathrm{RG}$ samples. The black traces are the absorption scans recorded after sample annealing to temperatures of 28,38 , and $60 \mathrm{~K}$ for $\mathrm{Ar}, \mathrm{Kr}$, and $\mathrm{Xe}$, respectively. Exciting into the absorption band maxima, at the indicated wavelengths, produces intense emission in each RG host as shown by the red traces. The position ${ }^{10}$ of the atomic barium $(6 \mathrm{~s} 6 \mathrm{p}){ }^{1} \mathrm{P}_{1} \leftarrow\left(6 \mathrm{~s}^{2}\right)^{1} \mathrm{~S}_{0}$ transition at $553.7 \mathrm{~nm}$ in the gas phase, is shown by the dashed vertical line, as is the $(6 s 6 \mathrm{p}){ }^{3} \mathrm{P}_{1} \leftarrow$ $\left(6 \mathrm{~s}^{2}\right)^{1} \mathrm{~S}_{0}$ transition at $791.35 \mathrm{~nm}$. The structures evident in the absorption spectrum of $\mathrm{Ba} / \mathrm{Xe}$ on both sides of the atomic ${ }^{1} \mathrm{P}_{1} \leftarrow{ }^{1} \mathrm{~S}_{0}$ band are due to barium dimer. Their concentration dependence and emission characteristics have been analyzed and presented in ref 4 for all three host solids.

analysis assumed the occurrence of a Jahn-Teller threefold split band for each matrix site, as was the case for the other spherically symmetric M/RG systems studied by the Maynooth Group. ${ }^{5,6}$ However, the present analysis of the (6s6p) ${ }^{1} \mathrm{P}_{1} \leftrightarrow\left(6 \mathrm{~s}^{2}\right)^{1} \mathrm{~S}_{0}$ luminescence of $\mathrm{Ba}$ isolated in the three RG solids (Ar, $\mathrm{Kr}$, and $\mathrm{Xe}$ ) reveals a more complex situation. Excitation spectroscopy was particularly useful in revealing bands severely overlapped in the absorption spectra, the details of which will now be presented.

The approach adopted in the present study to interpret the large variety of site-related excitation (absorption) bands relies on an observation first presented by Laursen and Cartland, ${ }^{7}$ who showed that the matrix shifts for atomic $\mathrm{P} \leftarrow \mathrm{S}$ absorptions of the group 12 metal atoms are approximately linear when plotted against the polarizability of the RG hosts. The basis for this simple dependence only became evident when the molecular constants of the 1:1 van der Waals complexes formed between the group 12 metal atoms $\mathrm{Zn}, \mathrm{Cd}$, and $\mathrm{Hg}$ and the heavier rare gas solids $\mathrm{Ar}, \mathrm{Kr}$, and $\mathrm{Xe}$ were obtained. All these complexes are now known ${ }^{8}$ to have relatively short $\mathrm{M}-\mathrm{RG}$ bond lengths that closely match the nearest neighbor distances in the rare gas solids. The good size match of the guest atoms and the host vacancies favors single substitutional site occupancy for all three metal atoms in the heavier rare gas solids, $\mathrm{Ar}-\mathrm{Xe}$. With the same (single vacancy) site occupied in these $\mathrm{M} / \mathrm{RG}$ systems, a linear dependence of matrix shift with host polarizability is observed. This behavior was also found for atomic $\mathrm{Na},{ }^{9} \mathrm{Mn},{ }^{5}$ and $\mathrm{Eu}^{6}$ even though considerable guest/host size mismatches exist, resulting in more complex absorption profiles from the occupancy of these guest atoms in multiple sites. In these cases, polarizability plots allowed for associations to be made for the same site types occupied by metal atoms in the three rare gas solids. Accordingly, matrix bands that exhibit a linear dependence of the matrix shift versus rare gas polarizability are expected for metal atoms trapped in a particular site type. On this basis, the same site occupied by atomic barium in the three solid rare gases can be identified from plots of the matrix shifts versus polarizabilities of the host solids.

State assigning the emission bands shown in Figure 1 is simple in that, based on their proximity to the gas phase position ${ }^{10}$ (indicated by the dashed vertical line), the observed emissions are naturally attributed to the $6 \mathrm{~s} 6 \mathrm{p}{ }^{1} \mathrm{P}_{1} \rightarrow 6 \mathrm{~s}^{2}{ }^{1} \mathrm{~S}_{0}$ transition of atomic Ba. Scans made out to $950 \mathrm{~nm}$ (the limit of our detection system) did not reveal any relaxed emission from the lower energy ${ }^{1} \mathrm{D}_{2}$ or ${ }^{3} \mathrm{P}_{J}$ states of atomic barium. Although our experimental setup does not allow detection of the ${ }^{3} \mathrm{D}_{1}$ state (which for the free atom occurs in the NIR at $1170 \mathrm{~nm}$ ), the absence of the nearby metastable states and the low intensity laser excitation used indicate negligible involvement of transitions from metastable levels. This observation is consistent with similar findings by Lebedev et al. ${ }^{11}$ for atomic barium isolated in solid, pressurized helium when resonant excitation at approximately $18500 \mathrm{~cm}^{-1}$ was used instead of high power radiation of the frequency-tripled $\mathrm{Nd}$ :YAG laser at $28195 \mathrm{~cm}^{-1}$. Under resonant excitation of $\mathrm{Ba} / \mathrm{He}$ with a weaker OPO laser, the strongest fluorescence observed was the line at $18115 \mathrm{~cm}^{-1}$ which corresponds to the transition from the $6 \mathrm{~s} 6 \mathrm{p}{ }^{1} \mathrm{P}_{1}$ state to the ground $6 \mathrm{~s}^{2}{ }^{1} \mathrm{~S}_{0}$ state. This is what we also observe with resonant excitation of atomic barium in the solid rare gases (SRGs) $\mathrm{Ar}, \mathrm{Kr}$, and $\mathrm{Xe}$ and accordingly assign to the $6 s 6 \mathrm{p}^{1} \mathrm{P}_{1} \rightarrow 6 \mathrm{~s}^{2}{ }^{1} \mathrm{~S}_{0}$ transition. This assignment is confirmed with excited state lifetime measurements conducted in the present study where nanosecond decay times were recorded in each solid.

In contrast to the relatively straightforward state assignment, the emission band profiles shown in Figure 1 suggest that the luminescence of $\mathrm{Ba}$ in these hosts is complex. In particular, the presence of multiple emission bands in the $\mathrm{Ba} / \mathrm{Ar}$ and $\mathrm{Ba} / \mathrm{Kr}$ systems points to a wider variety of site occupancy than in $\mathrm{Ba} /$ Xe. This situation is manifested also in the absorption bands shown in Figure 1. The detailed examination presented in this study relies on the excitation spectra recorded for the numerous distinct emission bands that exist in each host, to provide resolved bands which are strongly overlapping in absorption and whose origins are the multiple site occupancy of the barium atom. In a recent combined experimental and theoretical study of the $\mathrm{Ba} / \mathrm{Xe}$ system, ${ }^{1}$ the resolved excitation spectra of the thermally stable blue and violet bands were attributed to a four-atom tetravacancy (TV) and a five-atom vacancy, grain boundary site, respectively. The minor green band present in $\mathrm{Ba} / \mathrm{Xe}$ was attributed to a six-atom hexavacancy (HV) site. From a site occupancy perspective, $\mathrm{Ba} / \mathrm{Xe}$ is a simpler system than either of the lighter hosts and forms a basis for the discussion of the sites occupied by barium atoms in $\mathrm{Ar}$ and $\mathrm{Kr}$. The strength of the present study, in which the combined results of the three host solids are analyzed together, is that its overview provides greater scope to check the site proposals made recently in the $\mathrm{Ba} / \mathrm{Xe}$ system. ${ }^{1}$

The results are presented in the following manner. First, an illustration of the effects of reabsorption, as monitored with $2 \mathrm{D}$-EE spectroscopy, is provided using the $\mathrm{Ba} / \mathrm{Ar}$ system as a case study. Second, an overview of the entire $(6 s 6 \mathrm{p}){ }^{1} \mathrm{P}_{1}$ state 
luminescence of each $\mathrm{Ba} / \mathrm{RG}(\mathrm{RG}=\mathrm{Ar}, \mathrm{Kr}$, and $\mathrm{Xe})$ system is provided with the $2 \mathrm{D}$-EE spectra. Only a summary of the $\mathrm{Ba} /$ RG matrix spectra is presented here to allow comparisons to be made between the sites present in the three systems. Full spectroscopic details of the data recorded for the individual $\mathrm{Ba} / \mathrm{Ar}, \mathrm{Ba} / \mathrm{Kr}$, and $\mathrm{Ba} / \mathrm{Xe}$ matrices are provided elsewhere. ${ }^{12}$ Following this, steady state and time-resolved emission scans are presented for each of the distinct trapping sites identified. Emission scans and decay profiles are then recorded at elevated temperatures to investigate the existence of any nonradiative decay processes competing with the resonance fluorescence of $\mathrm{Ba}$ at a given site of isolation. Finally, a discussion of the possible sites occupied in the three $\mathrm{Ba} / \mathrm{RG}$ systems is provided based on the polarizability plots and the recent theoretical work done on the $\mathrm{Ba} / \mathrm{Xe}$ system. ${ }^{1}$

\section{METHODS}

All the data presented here were obtained, as outlined in the preceding papers, ${ }^{1,4}$ from samples deposited at $10 \mathrm{~K}$ under conditions of low metal loading which were subsequently annealed to minimum temperatures of 28,38 , and $60 \mathrm{~K}$ for Ar, $\mathrm{Kr}$, and $\mathrm{Xe}$, respectively. The experimental arrangement used to record luminescence is the same as that described previously for the $\mathrm{Ba} / \mathrm{Xe}^{1}$ system and expanded on below. The only significant difference is that extensive use was made of twodimensional excitation and emission (2D-EE) spectroscopy allowing emission bands arising from multiple site occupancy to be identified. These plots also allow for changes in the luminescence to be easily tracked, such as those arising from reabsorption effects and those that occur with matrix annealing. More significantly, the excitation traces extracted from carefully selected emission regions of the 2D-EE spectra are then used to deconvolute the absorption profiles into sitespecific features.

Emission spectra were recorded perpendicular to the excitation beam with an Acton Research $0.5 \mathrm{~m}$ SP500i monochromator using two distinct optical arrangements. ${ }^{1,12}$ For steady state work the dispersed output of a tungsten lamp and a Hamamatsu R928P PMT detector, maintained at -20 ${ }^{\circ} \mathrm{C}$ in a Products for Research (Photocool, S600) cooled housing, were used. For nanosecond time-resolved work an intensified, time-gated charge coupled device (iCCD) detector (Andor Technologies, model iStar DH720) held at $-15^{\circ} \mathrm{C}$ by an integrated Peltier cooling system was used with pulsed excitation from a Q-switched Nd:YAG laser (Quantel, YG980E-10). The third harmonic of the YAG $(355 \mathrm{~nm})$, operating at a repetition rate of $10 \mathrm{~Hz}$, was used to pump a dye laser (Quantel, TDL-90). Coumarin 540A dye was used to produce tunable laser radiation in the 523-586 nm range for pumping the resonance $6 \mathrm{~s} 6 \mathrm{p}{ }^{1} \mathrm{P}_{1} \leftarrow 6 \mathrm{~s}^{2}{ }^{1} \mathrm{~S}_{0}$ transition of atomic barium in the solid rare gases. Laser intensities were kept low, in the range $2-6 \mu \mathrm{J} / \mathrm{mm}^{2}$ (recorded at the sample window with a Molectron Power-max 500 A meter and PM10V1 head), to ensure one-photon transitions of the barium atoms. The excitation fluences were obtained using just the oscillator and/ or preamplifier of the dye laser and values varied in the range quoted, depending on the wavelength selected. The resulting emission was detected with the time-gated iCCD camera setting the observation interval to the minimum value of $2 \mathrm{~ns}$ and moving in $1 \mathrm{~ns}$ steps to the limit of where the emission intensity was detectable. This was less than $100 \mathrm{~ns}$ for all of the $\mathrm{Ba} / \mathrm{RG}$ systems. From the recorded time-resolved spectra, decay curves were extracted at selected emission wavelengths.
Excited state lifetimes were obtained by fitting exponential functions (after reconvolution with the laser pulse) to the recorded emission decay curves.

The iCCD detector was also used to record time-integrated (steady state) spectra by switching to its continuous-wave mode of operation. In this arrangement, the Andor iStar software and card (CCI-010), mounted on a PC, controlled the SP-500i monochromator to select the emission region of interest. For this setup the dispersed output of the tungsten lamp was used as the light source and by scanning the excitation monochromator-an ARC SP-300i-the total luminescences of the $\mathrm{Ba} / \mathrm{RG}$ matrices were recorded. These scans are presented in the form of $2 \mathrm{D}$-EE spectra. In all the 2D-EE spectra shown, scattered light from the excitation source has been removed for the purposes of presentation. Removal of the excitation light produces a well-defined diagonal border on the short wavelength side of the recorded emission as shown in Figures 2-4. The remainder of the scans presented in this work are from carefully annealed sampleshence the features shown are considered to arise from thermally stable sites.

\section{RESULTS AND DISCUSSION}

III.a. Reabsorption Effects. During the course of this work, it was noted that the OD of $\mathrm{Ba} / \mathrm{RG}$ samples greatly affected the appearance of the recorded luminescence. This effect is an optical artifact arising from strong reabsorption of the emission that occurs when there is extensive overlap with absorption bands of a fully allowed atomic resonance transition. The 2D-EE spectra presented in Figure 2, recorded in the region of the $(6 \mathrm{~s} 6 \mathrm{p}){ }^{1} \mathrm{P}_{1}$ luminescence of $\mathrm{Ba}$ isolated in solid Ar, illustrate this effect. The data shown in the panels were obtained from two samples both deposited at $10 \mathrm{~K}$, but with differing absorption strengths. The top spectrum corresponds to a sample with an optical density (OD) of 0.51 at $528 \mathrm{~nm}$, while the bottom spectrum was obtained from a less absorbing sample $(O D=0.28$ at $528 \mathrm{~nm}$ ). Three emission regions are observed in both spectra, centered at approximately 537,551 , and $562 \mathrm{~nm}$; however, the relative intensities of the three bands differ greatly depending on the OD of the sample. Thus, in the stronger absorbing sample the longest wavelength emission feature dominates the luminescence in agreement with the original $\mathrm{Ba} / \mathrm{Ar}$ spectra recorded by $\mathrm{B} \& \mathrm{~W}$, where only the $568 \mathrm{~nm}$ band was observed. In contrast, the emission band at $551 \mathrm{~nm}$ is the most intense in the less absorbing sample, consistent with the $\mathrm{Ba} / \mathrm{Ar}$ spectrum shown in Figure 1.

The effects of reabsorption can be reduced further by sample annealing as illustrated in Figures 3 and 4, which present the changes recorded in the $\mathrm{Ba} / \mathrm{Ar}$ and $\mathrm{Ba} / \mathrm{Kr}$ systems, respectively. It is clearly evident in the two figures that the red emission features are removed in both systems. In general, the shortest wavelength emission bands are more prominent and resolved in the weaker absorbing samples which have been annealed. This is a demonstration of the requirement for low optical densities to reduce reabsorption effects and thereby obtain the complete emission spectroscopy. The full extent of the spectral overlap between absorption and emission was illustrated in the recent $\mathrm{Ba} / \mathrm{Ar}$ work published by Fairbank and co-workers. ${ }^{3}$ Their Ba/Ar spectrum is similar to that shown in Figure 1, except the complete band profile of the $537 \mathrm{~nm}$ emission was not recorded in the earlier work. 

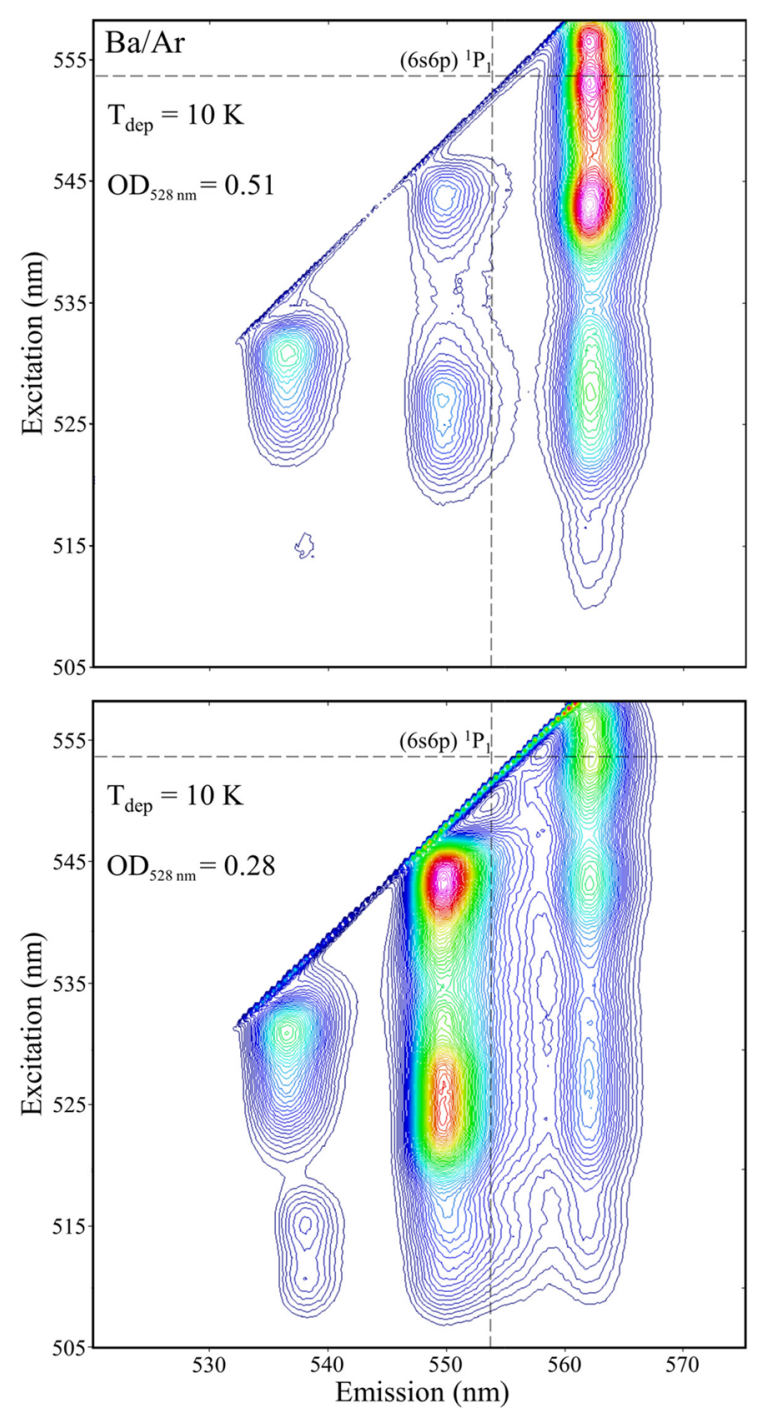

Figure 2. Two-dimensional excitation/emission (2D-EE) spectra recorded for the entire $(6 s 6 \mathrm{p}){ }^{1} \mathrm{P}_{1}$ state luminescence of $\mathrm{Ba}$ isolated in Ar illustrating the effects of optical density on the recorded spectra. The top panel depicts the luminescence originating from a sample with an absorption optical density (OD) of 0.51 at $528 \mathrm{~nm}$. The bottom spectrum corresponds to a less absorbing sample with an OD of 0.28 (at $528 \mathrm{~nm}$ ). Clearly the sample with the larger the $\mathrm{OD}_{528}$ produces much stronger red emission features with greatly diminished blue features compared with the $\mathrm{OD}=0.28$ sample. Both samples were deposited at $10 \mathrm{~K}$. The gas phase position ${ }^{10}$ of the $(6 \mathrm{~s} 6 \mathrm{p}){ }^{1} \mathrm{P}_{1} \leftarrow$ $\left(6 \mathrm{~s}^{2}\right)^{1} \mathrm{~S}_{0}$ transition of $\mathrm{Ba}$ at $553.7 \mathrm{~nm}$ is shown, in both excitation and emission, as dashed lines.

The origins of the effects of strong reabsorption are the multisite nature of the isolation of barium atoms which produce broad and complex $(6 \mathrm{~s} 6 \mathrm{p}){ }^{1} \mathrm{P}_{1}$ absorption profiles. These absorption profiles, presented in our earlier $\mathrm{Ba} / \mathrm{RG}$ work, ${ }^{4}$ were observed extending over the spectral range from 505 to $562 \mathrm{~nm}$. Accordingly, the $\mathrm{Ba} / \mathrm{Ar}$ emission bands centered at 537 and $551 \mathrm{~nm}$ which occur within this region are therefore very prone to competitive reabsorption. The extent of reabsorption, as shown in Figure 2, depends on the optical density of the doped RG solid. As a consequence, if a Ba/Ar sample is strongly absorbing, the lowest energy emission band dominates and the corresponding excitation spectrum will consist of a continuum spanning the entire absorption range. As each emission band corresponds to a unique site of isolation for $\mathrm{Ba}$ in solid $\mathrm{Ar}$, resolving site occupancy of the guest metal atom, under such conditions, will not be possible in the excitation spectra recorded. The situation described was also observed for $\mathrm{Ba} / \mathrm{Kr}$ following sample deposition at $10 \mathrm{~K}$. As was shown previously, ${ }^{1}$ this effect is not as significant for $\mathrm{Ba} /$ $\mathrm{Xe}$ as the dominant emission features are Stokes shifted away from the absorption profile and Xe contains fewer sites of isolation for atomic $\mathrm{Ba}$ than the two lighter hosts.

III.b. Site Association. Summaries of the complete excitation spectroscopy recorded for atomic barium isolated in $\mathrm{Ar}, \mathrm{Kr}$, and $\mathrm{Xe}$ matrices are presented in Figure 5 and contrasted with the absorption profiles shown by the broken black traces. The excitation scans were selected by identifying the unique emission bands (X.n) in the 2D-EE spectra shown in Figures 3 and 4 for Ar and $\mathrm{Kr}$ respectively and in Figure 1 of ref 1 for $\mathrm{Ba} / \mathrm{Xe}$. It is evident that these scans provide resolved bands which are severely overlapped in the absorption spectra. Indeed many of these bands are not evident in absorption, especially in the $\mathrm{Ba} / \mathrm{Ar}$ and $\mathrm{Ba} / \mathrm{Kr}$ systems. It is clear in Figure 5 that the $\mathrm{Ba} / \mathrm{Xe}$ system presents by far the simplest $\mathrm{Ba} / \mathrm{RG}$ matrix, exhibiting just two dominant sites of isolation. In addition, a minor "green" trapping site was also identified for atomic barium in xenon based on the distinct emission it produces. The comparison provided in Figure 5 indicates that the excitation spectra recorded for $\mathrm{Ba}$ in $\mathrm{Ar}$ and $\mathrm{Kr}$ present some features similar to those observed in Xe. Thus, in both Ar and $\mathrm{Kr}$ matrices, excitation bands associated with violet, blue, and green sites of isolation were observed. A fourth matrix "red" excitation profile was also observed to be stable in Ar and unstable in Kr. Although complex, the dominant components in the red excitation traces, in line shape and emission analyses to be shown below, were attributed to two distinct trapping sites (R1 and R2) for atomic Ba. For comparative purposes, the unstable red profile of $\mathrm{Ba} / \mathrm{Kr}$ is also included in the analysis.

The traces shown in Figure 5 for the three Ba/RG systems are color coded to match the site labeling scheme, whose origins are in the polarizability model-the details of which will now be presented. Gas phase to matrix frequency shifts are calculated for each of the three components of the blue, violet, and R1 (asymmetric) site bands. As the green site excitation spectra did not present any resolved structure, the band centers were used in lieu. However, as will be shown in Figure 8, the locations of the underlying three component bands were extracted in Gaussian line shape fits of the of the green site excitation profiles. Plots of these matrix shifts versus the host polarizability are presented in Figure 6.

From the approximately linear behavior exhibited, the polarizability analysis demonstrates a correspondence between the sites of a given "color" in all three hosts. For example, the dominant site in Xe, which shows a Jahn-Teller (JT) splitting (the blue trace in Figure 5), is associated with the JT band observed in $\mathrm{Kr}$ and the minor, poorly resolved threefold split band in Ar. Thus, it is proposed that each site "color" represents a particular type of trapping site for atomic barium in the rare gas solids. The details of the absorption/excitation bands unique to each site of isolation are presented in Table 1 for all three RG hosts. Interestingly, it is observed that the excitation components of a given site are not exactly linearly dependent on the host. For the violet, blue, and green sites, the slope of the line from $\mathrm{Ar}$ to $\mathrm{Kr}$ is greater than that from $\mathrm{Kr}$ to Xe. This highlights the simplicity of the polarizability model, which does not take into account the very different interactions 

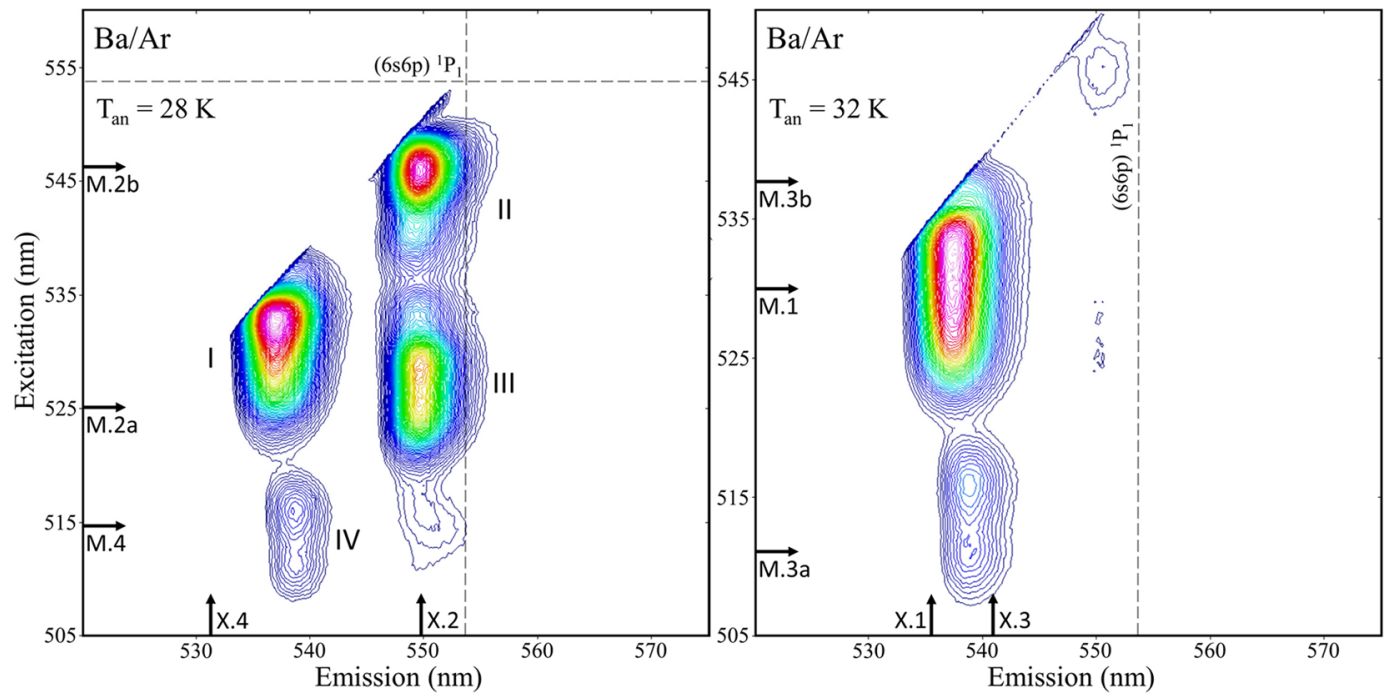

Figure 3. Two-dimensional excitation/emission spectra recorded for $(6 \mathrm{~s} 6 \mathrm{p}){ }^{1} \mathrm{P}_{1}$ state luminescence of $\mathrm{Ba} / \mathrm{Ar}$, following sample annealing. As indicated in the left panel, the emission band at approximately $562 \mathrm{~nm}$ has been completely removed at $28 \mathrm{~K}$ indicating its thermal instability, while the emission band at approximately $550 \mathrm{~nm}$ has almost been completely removed at $32 \mathrm{~K}$. By keeping the optical density low and with sample annealing, the blue emission features can be identified and their excitation spectra recorded.

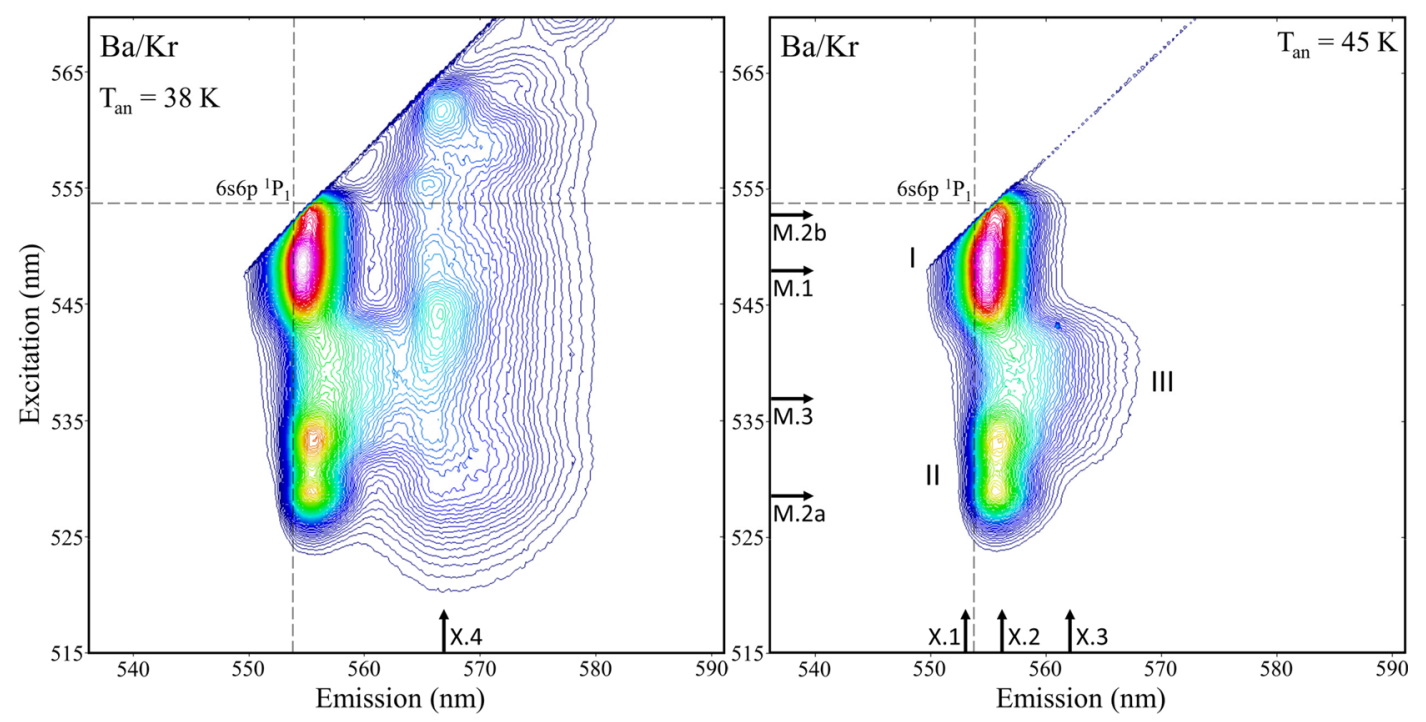

Figure 4. Effect of sample annealing on $\mathrm{Ba} / \mathrm{Kr}$ system as monitored by $2 \mathrm{D}$-EE spectra recorded for the $(6 s 6 \mathrm{p}){ }^{1} \mathrm{P}_{1}$ luminescence of atomic $\mathrm{Ba}$ isolated in krypton. As observed in the $\mathrm{Ba} / \mathrm{Ar}$ system, the red emission features are completely removed with annealing to $45 \mathrm{~K}$.

that occur between atomic barium and the surrounding RG environment in the ground, ${ }^{1} \mathrm{~S}_{0}$, and excited, ${ }^{1} \mathrm{P}_{1}$, states.

III.c. Blue Site Luminescence. A summary of the blue site luminescence recorded in the three $\mathrm{Ba} / \mathrm{RG}$ matrix systems is presented in Figure 7. The excitation traces of $\mathrm{Kr}$ and $\mathrm{Xe}$ both exhibit pronounced threefold splitting which is attributed to the Jahn-Teller (JT) effect, while the corresponding band in Ar seems to only show a partly resolved doublet. However, a line shape analysis conducted on this profile (shown by the three gray traces in Figure 7) reveals that a weak, higher energy band, located at approximately $513 \mathrm{~nm}$, makes up the third component of the characteristic JT pattern. Based on the band splitting exhibited, the blue site of isolation must be one of high symmetry $\left(O_{h}\right.$ or $\left.T_{d}\right)$, which gives rise to a dynamic Jahn-Teller distortion in the excited P state of atomic barium.

The abundance of the blue site varies considerably depending on the host. It is the dominant site of isolation for $\mathrm{Ba}$ in a Xe matrix, but in contrast, blue site occupation is reduced in $\mathrm{Kr}$ where the green and violet sites are similarly dominant. In $\mathrm{Ar}$, this site is so weakly populated that it is difficult to detect as revealed by the poor signal-to-noise ratio evident in the excitation scan shown in the top panel of Figure 7. It seems to only form in Ar samples deposited at higher temperatures (with a very low metal flux).

Inspection of Figure 7 reveals that the expected progressive red shift of the excitation/emission (ex/em) components of this site clearly occurs in $\mathrm{Kr}$ to $\mathrm{Xe}$ as one moves to a more polarizable RG host. The behavior in Ar is not immediately evident as the blue site emission is weak (indicated by the asterisk) due to the small amount of it present in this matrix and the threefold splitting pattern in excitation is not pronounced. However, with careful analysis, the ex/em components in $\mathrm{Ba} / \mathrm{Ar}$ have been identified. As indicated in Figure 7 , the ex/em bands of the $\mathrm{Ba} / \mathrm{Ar}$ system are located at 


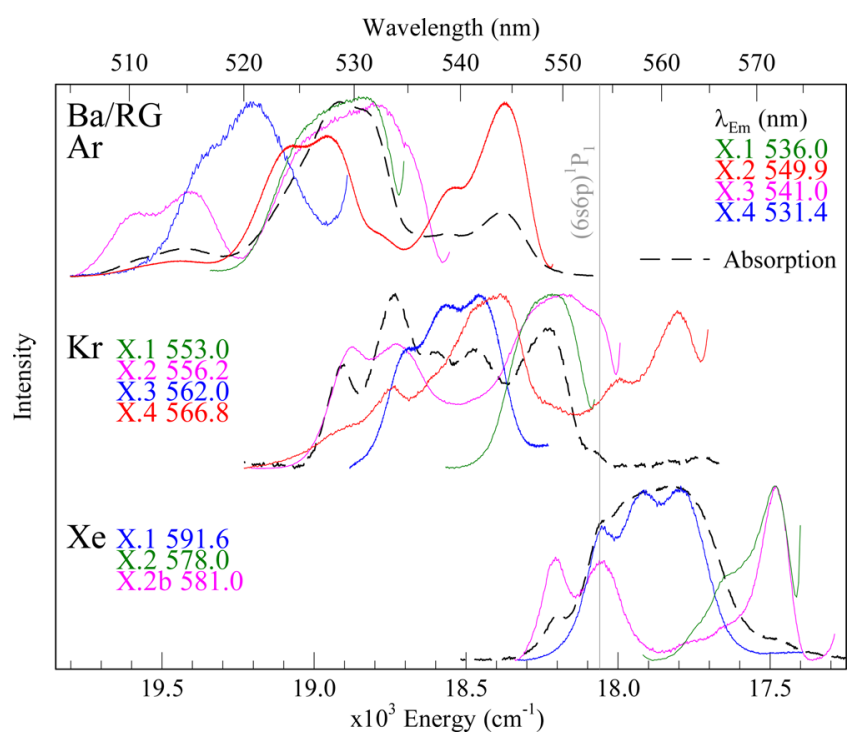

Figure 5. Site-specific $(6 s 6 \mathrm{p}){ }^{1} \mathrm{P}_{1} \leftarrow\left(6 s^{2}\right)^{1} \mathrm{~S}_{0}$ excitation spectra extracted at the indicated emission wavelengths (X.n) in the 2D-EE scans recorded for $\mathrm{Ba}$ atoms isolated in $\mathrm{Ar}, \mathrm{Kr}$, and Xe matrices at 10 $\mathrm{K}$. All the spectra shown were recorded following sample annealing. The absorption spectra of $\mathrm{Ba}$ (dashed black traces) are complicated by the presence of numerous distinct matrix sites which overlap considerably. The color of each excitation trace represents the site of isolation. Solid colored lines are used to present the excitation scans for the violet, blue, green, and red sites. For clarity, some of the excitation profiles have been truncated in an attempt to remove any overlapping components.

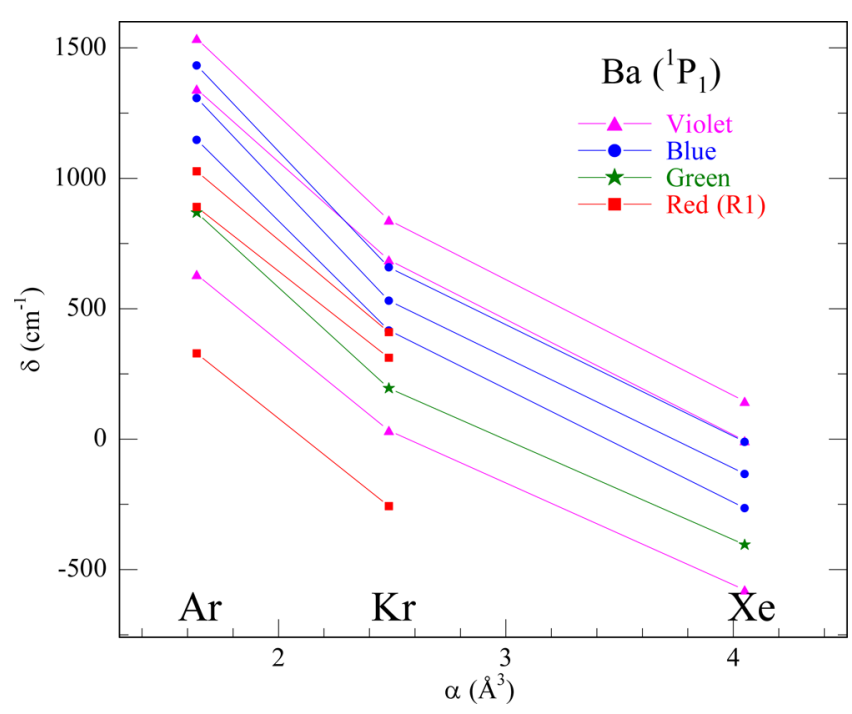

Figure 6. Plot of $\mathrm{Ba} / \mathrm{RG}$ matrix frequency shifts $\left(\delta, \mathrm{cm}^{-1}\right)$ relative to gas phase position of the $(6 \mathrm{~s} 6 \mathrm{p}){ }^{1} \mathrm{P}_{1} \leftarrow\left(6 \mathrm{~s}^{2}\right)^{1} \mathrm{~S}_{0}$ transition of atomic $\mathrm{Ba}$, observed for violet, blue, green, and red sites versus RG host polarizabilities $\left(\alpha, \AA^{3}\right)$.

the highest energy for the three rare gas hosts. Values of the matrix shifts are given in Table 2. The magnitude of the shift is related to the strength of the short-range $M-R G$ repulsive interaction in the excited $\mathrm{P}$ state and strongly depends on the size of the vacancy. For instance, the most polarizable host, Xe, has the largest lattice parameter and therefore has the largest blue site radius. This leads to less confinement of the excited $6 \mathrm{p}$ orbital of $\mathrm{Ba}$. The effects of increased host polarizability and a larger lattice vacancy both contribute to produce a moderate
Table 1. Photophysical Characteristics of Ba/RG Sites of Isolation As Revealed by Excitation Spectroscopy Recorded for the $(6 s 6 \mathrm{p}){ }^{1} \mathrm{P}_{1} \leftarrow{ }^{1} \mathrm{~S}_{0}\left(6 \mathrm{~s}^{2}\right)$ Transition $^{a}$

\begin{tabular}{|c|c|c|c|c|}
\hline $\mathrm{Ba} / \mathrm{RG}$ site & component & $\lambda_{\mathrm{ex}}(\mathrm{nm})$ & $\nu\left(\mathrm{cm}^{-1}\right)$ & $\delta\left(\mathrm{cm}^{-1}\right)$ \\
\hline \multicolumn{5}{|l|}{ argon } \\
\hline \multirow[t]{3}{*}{ violet } & 1 & 510.3 & 19596 & 1536 \\
\hline & 2 & 515.4 & 19402 & 1342 \\
\hline & 3 & 535.0 & 18692 & 631 \\
\hline \multirow[t]{3}{*}{ blue } & 1 & 513.0 & 19493 & 1433 \\
\hline & 2 & 516.3 & 19369 & 1308 \\
\hline & 3 & 520.6 & 19209 & 1148 \\
\hline green & 1 & 528.3 & 18929 & 868 \\
\hline \multirow[t]{3}{*}{ red } & 1 & 523.9 & 19088 & 1027 \\
\hline & 2 & 527.7 & 18950 & 890 \\
\hline & 3 & 543.8 & 18389 & 329 \\
\hline \multicolumn{5}{|l|}{ krypton } \\
\hline \multirow[t]{3}{*}{ violet } & 1 & 529.1 & 18900 & 840 \\
\hline & 2 & 533.4 & 18748 & 687 \\
\hline & 3 & 552.7 & 18093 & 33 \\
\hline \multirow[t]{3}{*}{ blue } & 1 & 534.2 & 18720 & 659 \\
\hline & 2 & 537.9 & 18591 & 531 \\
\hline & 3 & 541.2 & 18477 & 417 \\
\hline green & 1 & 547.8 & 18255 & 195 \\
\hline \multirow[t]{3}{*}{$\mathrm{red}^{b}$} & 1 & 541.4 & 18471 & 410 \\
\hline & 2 & 544.3 & 18372 & 312 \\
\hline & 3 & 561.7 & 17803 & -257 \\
\hline \multicolumn{5}{|l|}{ xenon } \\
\hline \multirow[t]{3}{*}{ violet } & 1 & 549.3 & 18205 & 145 \\
\hline & 2 & 553.9 & 18054 & -6 \\
\hline & 3 & 572.0 & 17483 & -578 \\
\hline \multirow[t]{3}{*}{ blue } & 1 & 554.0 & 18051 & -10 \\
\hline & 2 & 557.8 & 17928 & -133 \\
\hline & 3 & 561.9 & 17797 & -264 \\
\hline green & 1 & 566.4 & 17655 & -405 \\
\hline
\end{tabular}

${ }^{a} \delta$ represents the gas phase to RG matrix shift (given in units of wavenumber) calculated from the gas phase value ${ }^{10}$ for this transition at $18060.261 \mathrm{~cm}^{-1}(553.7 \mathrm{~nm})$. The wavelength uncertainties in the excitation values are estimated to be $<0.1 \mathrm{~nm}$. In the working spectral range this translates into an uncertainty of $\pm 4 \mathrm{~cm}^{-1}$. ${ }^{b}$ The red site in $\mathrm{Kr}$ was shown to be a thermally unstable site of isolation but is included to allow comparison with thermally stable red site in Ar.

gas-to-matrix red shift of $-133 \mathrm{~cm}^{-1}$ for the blue site in Xe. Contrasting this, solid $\mathrm{Ar}$ is the least polarizable of the three hosts and also has the smallest lattice parameter. Therefore, the blue site radius in Ar will be smaller than in Xe and the excited $6 \mathrm{p}$ orbital of $\mathrm{Ba}$ will be expected to experience a greater repulsive interaction. The combination of both effects yields a large blue shift of $+1308 \mathrm{~cm}^{-1}$ (measured from the band center) for the blue site in Ar.

The photophysical characteristics of the site-specific fluorescence bands in the three $\mathrm{Ba} / \mathrm{RG}$ systems are collected in Table 2. Clearly the Stokes shift (SS) of the blue site emission, computed from the central component of the JT excitation band in each host, increases from Ar to Xe. Its value in the heaviest host $\left(\mathrm{Xe}, 1024 \mathrm{~cm}^{-1}\right)$ is almost twice that measured for the lightest (Ar, $\left.543 \mathrm{~cm}^{-1}\right)$. This difference is graphically illustrated in Figure 7 by the length of the "SS lines" shown there. In addition, the emission bandwidths (full width at half-maximum, fwhm) are also observed to increase from $\mathrm{Ar}$ to Xe. Both of these effects indicate that the minimum of the excited $\mathrm{Ba}\left({ }^{1} \mathrm{P}_{1}\right) / \mathrm{Xe}$ potential energy surface is displaced, with 


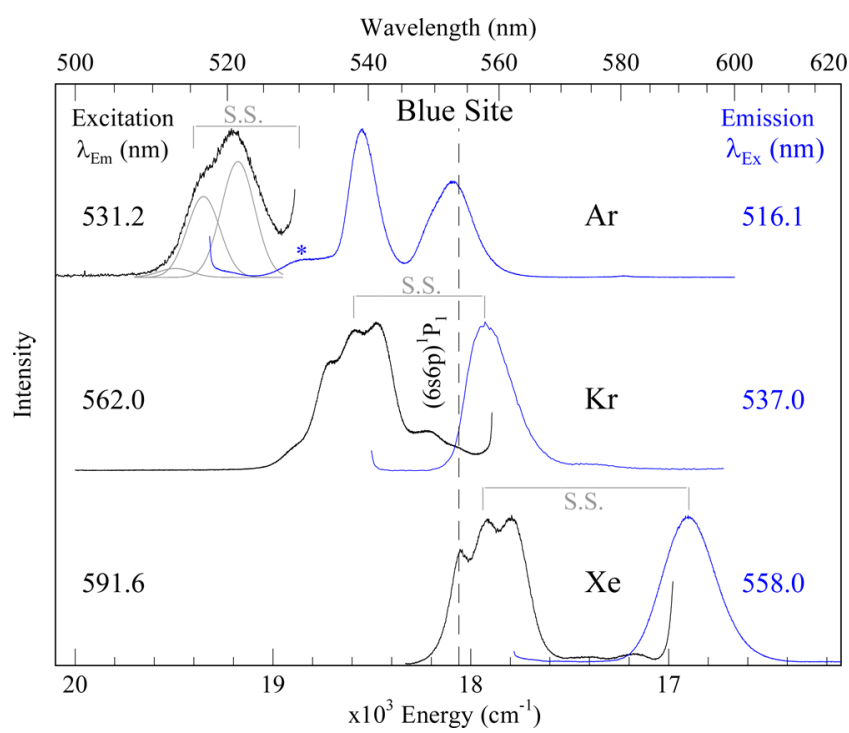

Figure 7. Summary of site-selected excitation (black) and emission spectra (blue traces) recorded for $\mathrm{Ba}$ atoms isolated in the blue site in the three rare gas solids studied in the present work. Threefold splitting is very evident in the $\mathrm{Xe}$ and $\mathrm{Kr}$ excitation spectra and not so in $\mathrm{Ar}$; the presence of three components in the blue site excitation spectrum in this solid was revealed in the Gaussian fit shown by the gray solid traces. The location of the weak blue-site emission in $\mathrm{Ba} /$ $\mathrm{Ar}$, due to the low occurrence of this site, is indicated by the asterisk. The horizontal lines labeled "S.S." show the excitation and emission values used to calculate the blue site Stokes shifts in each host. Each $\mathrm{RG}$ matrix has been annealed to 34,45 , and $60 \mathrm{~K}$ for $\mathrm{Ar}, \mathrm{Kr}$, and $\mathrm{Xe}$, respectively.

respect to minimum of the ground state, to a greater extent when compared to the $\mathrm{Ba} / \mathrm{Ar}$ and $\mathrm{Ba} / \mathrm{Kr}$ systems.

III.d. Green Site Luminescence. A summary of the luminescence specific to the green site of isolation is provided in Figure 8. The preference for the occupancy of atomic barium in the green site in the three rare gases matrices is the opposite of that of the blue site. In $\mathrm{Ar}$, the green site is the dominant trapping environment. In $\mathrm{Kr}$, this dominance is matched by both the violet and blue sites, while in $\mathrm{Xe}$, the absorption of the green site is much weaker.

Examination of the green site excitation profiles in $\mathrm{Ar}$ and $\mathrm{Kr}$ reveals, as highlighted in red in Figure 8, an asymmetric band shape that tails in both cases to higher energy with decreasing intensity. As splitting patterns are not obvious in any of the excitation profiles, the underlying structures of these asymmetric bands were extracted in line shape analyses. Three Gaussian curves (shown in gray in Figure 8) of equal width were required to adequately fit the profiles in $\mathrm{Ar}$ and $\mathrm{Kr}$. It is likely that these bands are broadened by the dynamic Jahn-Teller effect and the constituent peaks are unresolved. It is thereby proposed that the site of occupation is isotropic (or at least not far from it), perhaps one of high (cubic) symmetry given the nature of the ground state barium atom. If this is the case, then the green site of isolation must exhibit a larger radius than the blue site in each solid. Inspection of Table 1 reveals that the gas-to-matrix blue shifts for the green site in $\mathrm{Ar}$ and $\mathrm{Kr}$ are much less than those of the blue site. In addition, the partially resolved band in $\mathrm{Xe}$ is more red-shifted than the corresponding blue site in this host. Thus, the confinement of the excited $6 \mathrm{p}$ orbitals must be less significant for $\mathrm{Ba}$ isolated in the green site. The matrix shifts observed therefore depend more on the RG polarizability, leading to a red shift of the band center with respect to absorptions of the blue site.

Consistent with the confinement argument just presented, inspection of Table 2 reveals that the calculated Stokes shifts for this site are much less than those recorded for the blue site in each RG host. Moreover, the magnitude of the shift does not increase systematically across the three hosts, with values of 321,234 , and $327 \mathrm{~cm}^{-1}$ measured for $\mathrm{Ar}, \mathrm{Kr}$, and $\mathrm{Xe}$, respectively. This is in contrast to the trend observed for the blue site. The green site in $\mathrm{Kr}$ shows the smallest Stokes shift and emission bandwidth of the site systems identified in the three hosts. Overall, the emission bandwidths are noticeably narrower than those of the blue site. The combination of small Stokes shifts and narrow emission bandwidths indicates that the equilibrium configuration of the barium atom in the green site is nearly the same in the ground and excited states. Thus, a "vertical" Franck-Condon transition in absorption prepares the excited $\mathrm{Ba}$ atom in a configuration which is relatively close to the minimum of the excited state potential energy surface.

III.e. Violet Site Luminescence. A summary of the $\mathrm{Ba} /$ RG violet site luminescence is provided in Figure 9, which reveals a splitting pattern in excitation that is in stark contrast to the familiar threefold split JT pattern exhibited on $\mathrm{P} \leftarrow \mathrm{S}$ transitions of most matrix-isolated atoms. The classical JT structure is most clearly presented in the $\mathrm{Ba} / \mathrm{RG}$ system by the blue site. Contrasting with this, the excitation profiles of the $\mathrm{Ba} / \mathrm{RG}$ violet sites display an asymmetric $2+1$ splitting pattern, with the doublet at higher energy and a lower energy singlet. Each component peak of this $2+1$ structure is clearly identifiable in the $\mathrm{Ba} / \mathrm{Xe}$ system as shown in the bottom of Figure 9. In contrast, only the higher energy doublets are immediately evident in $\mathrm{Ar}$ and $\mathrm{Kr}$. For both $\mathrm{Ba} / \mathrm{Ar}$ and $\mathrm{Ba} / \mathrm{Kr}$ the singlet band is strongly overlapped by the green site absorption band. As a result, the location of the singlet component was identified in the line shape fits presented by the gray traces in Figure 9. The singlet band centers are shown by the short red vertical lines.

Examination of Figure 9 and Table 1 indicates that this site of isolation presents two very different interactions for the Ba atom excited into its $6 \mathrm{~s} 6 \mathrm{p}$ configuration. The splitting between the doublet and singlet features is substantial in each solid. Values of 794, 711, and $633 \mathrm{~cm}^{-1}$ are determined (from the lowest energy singlet to the center of the doublet) in $\mathrm{Ar}, \mathrm{Kr}$, and $\mathrm{Xe}$, respectively. This data may be interpreted by considering the orientation of the $6 \mathrm{p}_{x}, 6 \mathrm{p}_{y}$, and $6 \mathrm{p}_{z}$ orbitals of the excited $\mathrm{Ba}$ atom in the environment of the violet site. Evidently the interaction in this environment is anisotropic, such that two of the $\mathrm{p}$ orbitals $\left(\mathrm{p}_{x}\right.$ and $\left.\mathrm{p}_{y}\right)$ are pointing toward one or more RG atoms ("equatorial", $\Pi$ configuration) and thereby experience a strong repulsion. It is of note that the interaction of these two orbitals is slightly different, giving rise to a nondegenerate $\Pi$ doublet in absorption. Contrasting this, the $\mathrm{p}_{z}$ orbital must be directed toward more empty space ("polar", $\Sigma$ configuration) and experiences less repulsion with the host atoms. Thus, the higher energy doublet corresponds to the more repulsive $\Pi$ configuration and the lower energy singlet represents the $\Sigma$ configuration. Such a $\Sigma-\Pi$ splitting was also observed for the excited $\mathrm{Ba}\left({ }^{1} \mathrm{P}\right)$ atom on the surface of an Ar cluster. ${ }^{13}$ However, in that case, the $\Pi$ doublet was lower in energy than the $\Sigma$ singlet. The $6 \mathrm{p}_{z}$ orbital of Ba was directed toward the Ar cluster and $6 \mathrm{p}_{x}$ and $6 \mathrm{p}_{y}$ orbitals were aligned parallel to the cluster surface, pointing toward empty space. The asymmetric threefold $(2+1)$ splitting pattern 
Table 2. Photophysical Characteristics of Ba/RG Sites of Isolation As Revealed by $(6 s 6 \mathrm{p}){ }^{1} \mathrm{P}_{1} \rightarrow{ }^{1} \mathrm{~S}_{0}\left(6 \mathrm{~s}^{2}\right)$ Emission Spectroscopy ${ }^{a}$

\begin{tabular}{|c|c|c|c|c|c|c|}
\hline $\mathrm{Sr} / \mathrm{RG}$ site & $\lambda_{\mathrm{ex}}(\mathrm{nm})$ & $\lambda_{\mathrm{em}}(\mathrm{nm})$ & $\nu\left(\mathrm{cm}^{-1}\right)$ & $\delta\left(\mathrm{cm}^{-1}\right)$ & SS $\left(\mathrm{cm}^{-1}\right)$ & $\Delta \nu\left(\mathrm{cm}^{-1}\right)$ \\
\hline \multicolumn{7}{|l|}{ argon } \\
\hline \multirow[t]{3}{*}{ violet } & 510.3 & \multirow{3}{*}{539.1} & \multirow{3}{*}{18549} & & 1047 & \multirow{3}{*}{163} \\
\hline & 515.4 & & & 489 & 853 & \\
\hline & 535.0 & & & & 142 & \\
\hline blue & 516.3 & 531.2 & 18825 & 765 & 543 & $225^{b}$ \\
\hline green & 528.3 & 537.4 & 18608 & 548 & 321 & 183 \\
\hline \multirow[t]{3}{*}{ red } & 523.9 & \multirow{3}{*}{549.9} & \multirow{3}{*}{18185} & & 902 & \multirow{3}{*}{163} \\
\hline & 527.7 & & & 125 & 765 & \\
\hline & 543.8 & & & & 204 & \\
\hline \multicolumn{7}{|l|}{ krypton } \\
\hline \multirow[t]{3}{*}{ violet } & 529.1 & \multirow{3}{*}{556.2} & \multirow{3}{*}{17979} & \multirow{3}{*}{-81} & 921 & \multirow{3}{*}{129} \\
\hline & 533.4 & & & & 769 & \\
\hline & 552.7 & & & & 114 & \\
\hline blue & 537.9 & 557.9 & 17924 & -136 & 666 & 261 \\
\hline green & 547.8 & 554.9 & 18021 & -39 & 234 & 135 \\
\hline \multirow[t]{3}{*}{$\mathrm{red}^{c}$} & 541.4 & \multirow{3}{*}{567.0} & \multirow{3}{*}{17643} & \multirow{3}{*}{-417} & 828 & \multirow{3}{*}{$200^{b}$} \\
\hline & 544.3 & & & & 729 & \\
\hline & 561.7 & & & & 160 & \\
\hline \multicolumn{7}{|l|}{ xenon } \\
\hline \multirow[t]{3}{*}{ violet } & 549.3 & \multirow{3}{*}{578.1} & \multirow{3}{*}{17298} & \multirow{3}{*}{-762} & 907 & \multirow{3}{*}{213} \\
\hline & 553.9 & & & & 756 & \\
\hline & 572.0 & & & & 184 & \\
\hline blue & 557.8 & 591.6 & 16903 & -1157 & 1024 & 311 \\
\hline green & 566.4 & 577.1 & 17328 & -732 & 327 & 202 \\
\hline
\end{tabular}

${ }^{a} \delta$ represents the gas phase to RG matrix shift calculated from the gas phase value ${ }^{10}$ for this transition at $553.7 \mathrm{~nm}\left(18060.261 \mathrm{~cm}^{-1}\right) . \mathrm{SS}$ represents the Stokes shift. For the blue and green sites, this value was determined from the center of the emission band to the center of the excitation band. For the violet and red sites, three values are quoted which were evaluated from the center of the emission band to each excitation component of the asymmetric threefold split. The last column, $\Delta \nu$, gives the full width at half-maximum values for each emission band in units of wavenumber $\left(\mathrm{cm}^{-1}\right)$. The wavelength uncertainties in the emission values are estimated to be $<0.06 \mathrm{~nm}$. In this spectral range this translates into an uncertainty of $\pm 2 \mathrm{~cm}^{-1}$. ${ }^{b}$ The spectral bandwidths of the blue site emission in Ar and the R1 site in Kr were estimated with a line shape analysis as significant overlap with emission from other sites occurred in both cases. ${ }^{c}$ Thermally unstable site of isolation.

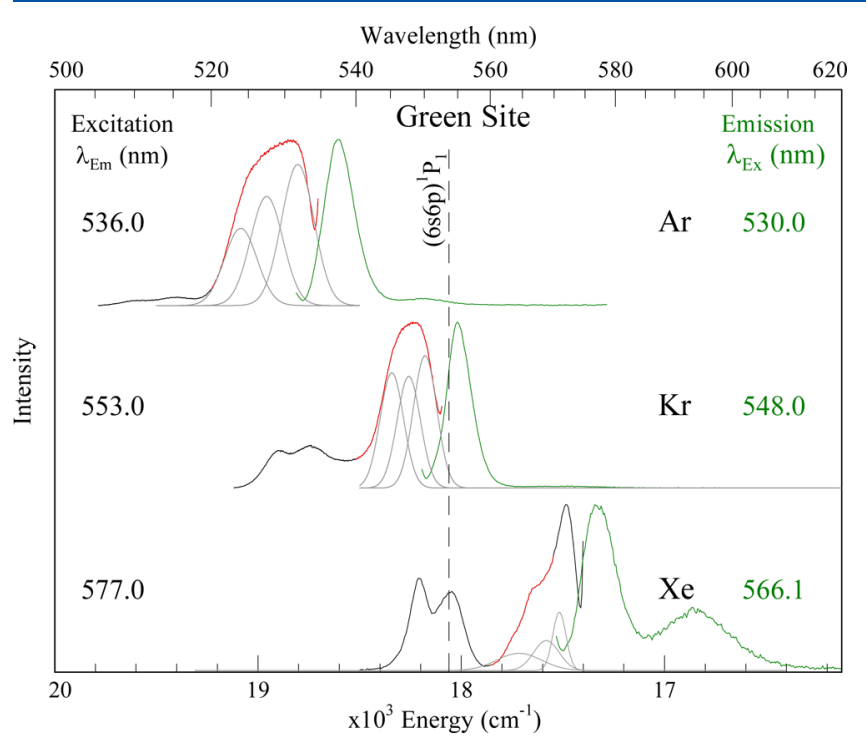

Figure 8. Summary of excitation (red) and emission spectra (green traces) recorded for $\mathrm{Ba}$ isolated in the green site of the RG solids. The three Gaussian curves used to fit the green site excitation band in Ar and $\mathrm{Kr}$ are shown by the solid gray traces. For clarity, the portion of each excitation band associated with the green site of isolation is highlighted in red. This band is especially weak in $\mathrm{Ba} / \mathrm{Xe}$ due to the low occurrence of this site in xenon.

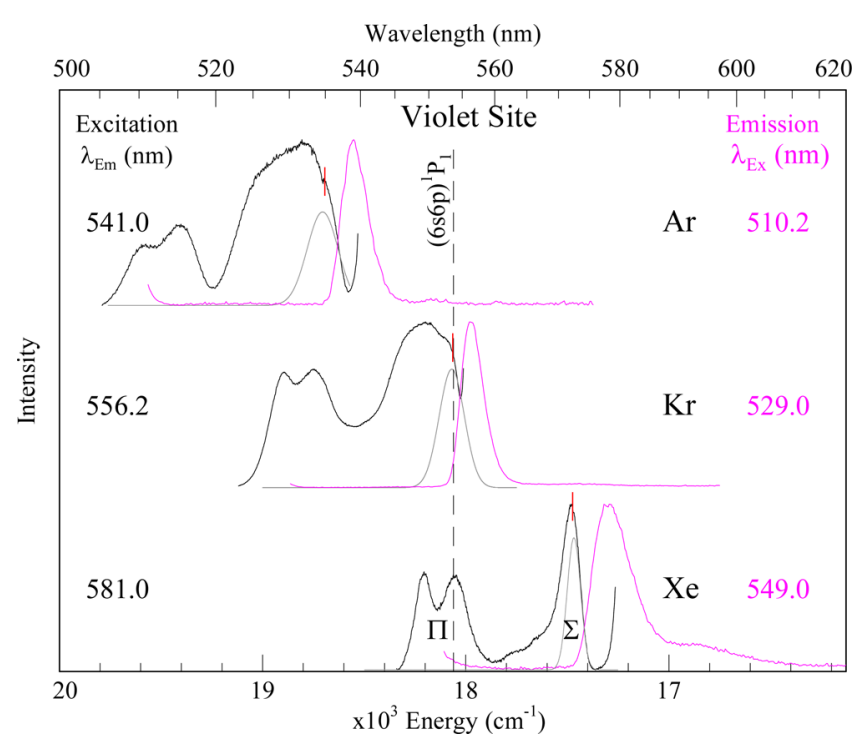

Figure 9. Summary of excitation (black) and emission spectra (purple traces) recorded for atomic barium isolated in the violet site in each RG solid. The Gaussian peaks (shown in gray) indicate the position of the lowest energy component of the asymmetric $2+1$ split band. Although the position of this band is obvious in $\mathrm{Xe}$, the corresponding components in $\mathrm{Ar}$ and $\mathrm{Kr}$ are obscured by an intense green site absorption. For clarity the position of this band is shown by vertical red lines for these two solids. 
observed for $\mathrm{Ba}$ in the RG solids suggests that the violet site of isolation corresponds to a highly axial site. Such a vacancy could be described in a generic sense as a "cylindrical" site. The fcc void that most closely matches this description is a divacancy, but as was found in the recent molecular dynamics (MD) calculations ${ }^{1}$ on $\mathrm{Ba} / \mathrm{Xe}$, this site is too small even in xenon to accommodate the large $6 \mathrm{~s}^{2}$ metal atom. The issue of site attributions will be made in section III.h.

In all three hosts, rapid (subnanosecond) nonradiative relaxation occurs to the lowest energy $\Sigma$ configuration, as only a single emission band is observed regardless of which of the 2 +1 components is photoexcited. The details of the Stokes shifts computed from the violet site $\Pi$ and $\Sigma$ bands in each host are presented in Table 2. Examination of these values demonstrates that a much larger Stokes shift occurs with excitation of the $\Pi$ bands-more than 8 times the value of the $\Sigma$ Stokes shift in the case of $\mathrm{Kr}$. Thus, following excitation of an electron into one of the $\Pi$ orbitals, a reorientation occurs along the excited state surface to minimize the initial strongly repulsive interactions and thereby lower the energy. From the $\Sigma$ orientation, the SS values are small, having values of only 142,114 , and $184 \mathrm{~cm}^{-1}$ respectively in $\mathrm{Ar}, \mathrm{Kr}$, and $\mathrm{Xe}$. The violet site excited state lifetime measurements conducted in $\mathrm{Xe}$ show the same nanosecond decay profile, regardless of the porbital orientation reached in the excited state. This behavior indicates that orbital reorientation occurs on a subnanosecond time scale.

III.f. Red Site Luminescence. A summary of the red site luminescence features in $\mathrm{Ar}$ and $\mathrm{Kr}$ is presented in Figure 10, and the photophysical characteristics of this site are collected in Tables 1 and 2. A detailed analysis, which included measuring emissions lifetimes and their temperature dependence, was conducted for $\mathrm{Ba} / \mathrm{Ar} .{ }^{12}$ The same extensive study

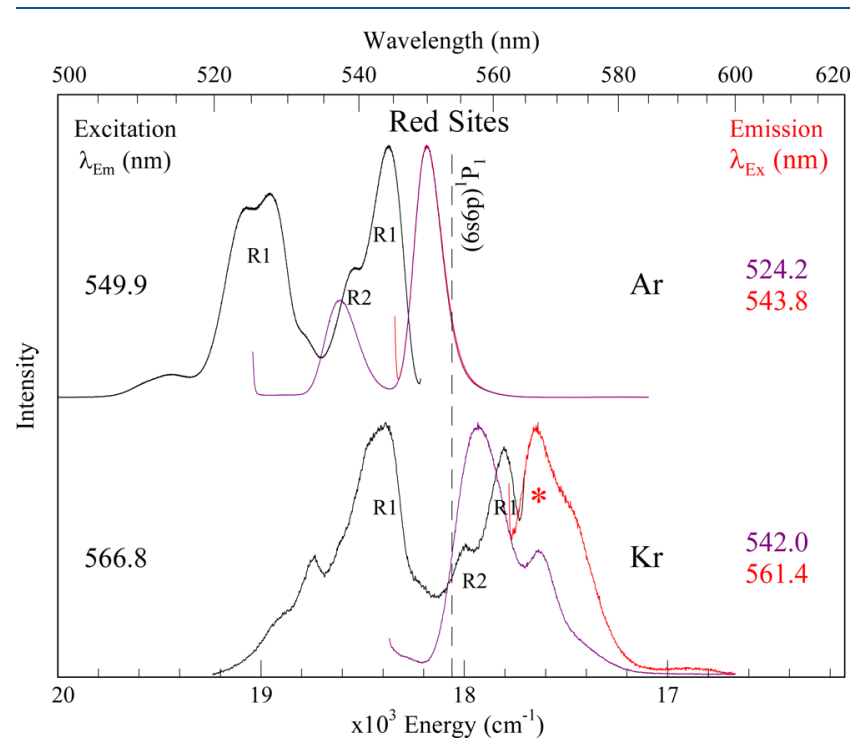

Figure 10. Summary of $(6 s 6 p){ }^{1} \mathrm{P}_{1}$ state luminescence of Ba isolated in the red site in solid $\mathrm{Ar}$ and $\mathrm{Kr}$ samples which were annealed to 28 and $38 \mathrm{~K}$, respectively. The red site in $\mathrm{Kr}$ was shown to be thermally unstable with annealing to $45 \mathrm{~K}$. The same site in $\mathrm{Ar}$ can be removed by annealing to $34 \mathrm{~K}$-with a substantial loss of the sample. As indicated in the upper portion of the figure, excitation into R1 at two different locations ( 543.8 and $524 \mathrm{~nm}$ ) produces the same emission centered at $549.9 \mathrm{~nm}$. In contrast, excitation into R2 at $538.9 \mathrm{~nm}$ produces distinct emission centered at $549.0 \mathrm{~nm}$ with a larger bandwidth. could not, however, be achieved for the equivalent bands in $\mathrm{Kr}$ owing to the thermal instability of this site in $\mathrm{Kr}$. Moreover, numerous distinct lower energy bands complicate the 2D-EE spectrum (see Figure 4) of a $\mathrm{Kr}$ sample annealed to $38 \mathrm{~K}$ indicating that a multitude of partially stable sites exist. For comparative purposes the excitation profile of the brightest emission in this region is presented in the bottom panel of Figure 10.

Even though the red-site emission in $\mathrm{Ba} / \mathrm{Ar}$ appears simple, its excitation profile is complex. From carefully monitoring the emission and after conducting fits of the excitation profile, it is proposed to consist of two distinct matrix sites, designated R1 and R2, and whose locations are indicated in Figure 10. Line shape fits of the $\mathrm{Ba} / \mathrm{Ar}$ red site excitation profile required the use of six Gaussian functions, the results of which are shown in Figure 11. Even though only five features are evident in the

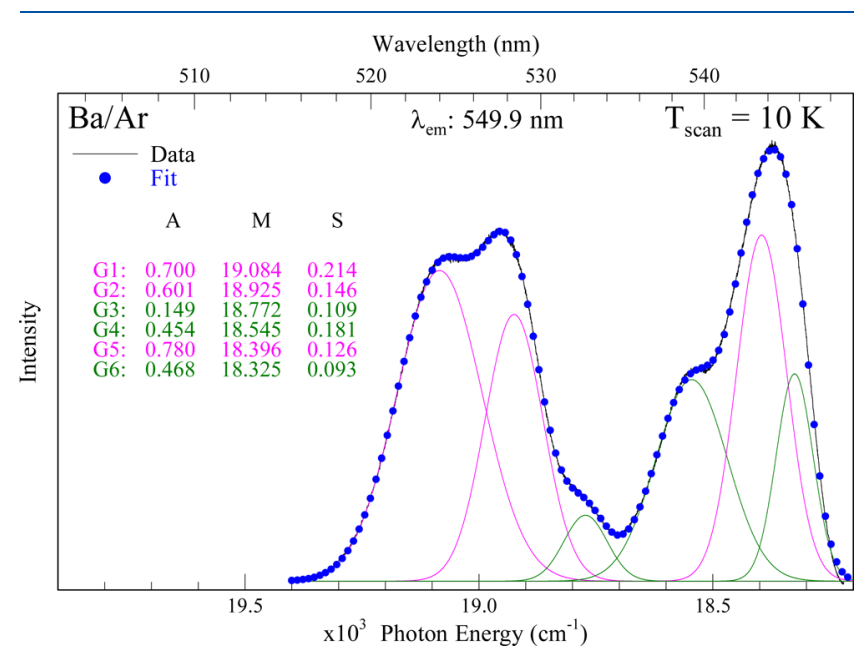

Figure 11. Line shape analysis conducted on the dominant, red site of isolation of atomic barium isolated in solid argon. Even though only five features are evident in the red site excitation profile, acceptable fits required the use of six Gaussian functions $(\mathrm{G} n)$. The parameters of the six functions are listed as $A, M$, and $S$-the amplitude, band position, and bandwidth $\left(\times 10^{3} \mathrm{~cm}^{-1}\right)$, respectively. The six curves are color coded revealing the presence of an asymmetric $2+1, \mathrm{R} 1$ (pink) band and a symmetric threefold split, R2 (green) band. The position of the lowest energy component of the R2 band is not obvious in the recorded profile but is essential to obtain an acceptable fit. On the basis of the fit shown, the $\mathrm{R} 1$ feature has an a symmetric $2+1$ splitting while the $\mathrm{R} 2$ is a more standard Jahn-Teller structured band.

recorded excitation profile, the best fits consistently indicated the existence of an additional, unresolved band (the lowest energy component) at $18325 \mathrm{~cm}^{-1}$. On the basis of the spectral locations and their relative intensities, the six components are grouped as two sets of bands-consisting of the asymmetric $2+1$ bands (R1 feature) and a more typical, JT threefold-split (R2) band. The latter extensively overlaps the lowest energy component of the $2+1$ band so that a clear distinction of their emission cannot be provided. However, as indicated in the upper portion of Figure 11, excitation into R1 at two different locations ( 543.8 and $524 \mathrm{~nm}$ ) produces the same emission centered at $549.9 \mathrm{~nm}$. In contrast, excitation into $\mathrm{R} 2$ at $538.9 \mathrm{~nm}$ produces distinct emission centered at $549.0 \mathrm{~nm}$ with a larger bandwidth.

Analysis of the luminescence in Ar suggests that the primary contributor is a trapping site (R1) which gives rise to a "doublet plus singlet" splitting pattern, similar to the violet site. 
A comparison with the thermally unstable red site profile of $\mathrm{Ba} / \mathrm{Kr}$ in Figure 10 (lower panel) reveals the same general features. The equivalent of the Ar higher energy doublet centered at $\sim 543 \mathrm{~nm}$ is not resolved in $\mathrm{Kr}$ but does exhibit a dominant peak at $544.3 \mathrm{~nm}$ and a shoulder at $541.4 \mathrm{~nm}$. The band at 555.8 in $\mathrm{Kr}$ is tentatively assigned as a secondary, minor red site (R2) based on the distinct emission produced at this excitation wavelength, as can be seen in the 2D-EE spectrum presented in Figure 4 (left panel).

On the basis of this interpretation, the primary R1 site of isolation gives rise to two distinct interactions for the $\mathrm{Ba}$ atom excited into the $(6 s 6 \mathrm{p}){ }^{1} \mathrm{P}_{1}$ state. A large splitting between the doublet and singlet feature is observed in $\mathrm{Ar}$ and $\mathrm{Kr}$. Values of 699 and $619 \mathrm{~cm}^{-1}$ are determined (from the lowest energy singlet to the center of the doublet) in $\mathrm{Ar}$ and $\mathrm{Kr}$ respectively-noticeably smaller than those obtained for the violet site. The splitting may be explained by considering the orientation of the $6 \mathrm{p}_{x}, 6 \mathrm{p}_{y}$, and $6 \mathrm{p}_{z}$ orbitals of the excited $\mathrm{Ba}$ atom with respect to the anisotropic configuration of the surrounding RG atoms. Similar to the violet site, the higher energy doublet corresponds to the more repulsive $\Pi$ configuration $\left(\mathrm{p}_{x}\right.$ and $\left.\mathrm{p}_{y}\right)$ and the lower energy singlet represents the $\Sigma$ configuration $\left(\mathrm{p}_{z}\right)$. It is interesting to note that the extent of splitting on the $\Pi$ doublet is less than that of the violet site. Combining this characteristic with a smaller overall singlet to doublet energy gap suggests a weaker interaction of the excited $\mathrm{Ba}$ atom at this site in comparison to the violet site. A more voluminous cylindrical site could therefore be proposed for R1. As the features of R1 are connected to a single emission band in $\mathrm{Ar}$ and $\mathrm{Kr}$, a relaxation to the lowest energy excited $(6 s 6 \mathrm{p}){ }^{1} \mathrm{P}_{1}$ state surface must occur in both hosts.

III.g. Temporal Analysis. Time-resolved emission spectra (TRES) were recorded, as described in the Methods section and elsewhere, ${ }^{1,12}$ with nanosecond pulsed laser excitation and time-gated iCCD detection. The decay profiles extracted were, in most cases, well-fitted with single exponential functionstypical data and fits were shown in the recent $\mathrm{Ba} / \mathrm{Xe}$ study. ${ }^{1}$ This allowed the excited state lifetimes to be determined, and the observed values $\left(\tau_{\text {obs }}\right)$ for each site of isolation in the three $\mathrm{Ba} / \mathrm{RG}$ systems are collected in Table 3 . With the exception of the blue site in $\mathrm{Ba} / \mathrm{Xe}$, all measured decay times were found to be invariant over a temperature range of at least $3 \mathrm{~K}$, indicating that the radiative lifetimes had been identified. The lifetime values are plotted in Figure 12 along with a $\lambda^{3}$ extrapolation of the gas phase ${ }^{10}$ value of 8.4 ns. As shown, the directly measured $\left(\tau_{\mathrm{obs}}\right)$ matrix values are all less than the gas phase extrapolation shown by the solid curve. However, in a comparison with the gas phase values, a correction must be made for the effective field of the solid. ${ }^{8}$ As described in a previous study on the $\mathrm{Zn} / \mathrm{RG}$ systems, ${ }^{14}$ this leads to an enhancement of electronic transitions and a shortening of the observed matrix lifetimes. The correction ${ }^{15,16}$ for the effective field of the solid host is made using the following equation:

$$
\tau_{\mathrm{cor}}=\tau_{\mathrm{obs}} n\left[s\left(n^{2}-1\right)+1\right]^{2}
$$

Equation $\mathrm{I}$ is a function of the index of refraction, ${ }^{17} n$, of the rare gas host and a "shape" parameter, s. The latter is a depolarization factor related to the symmetry of the site accommodating the guest atom. For an isotropic, spherical cavity $s=1 / 3$. However, this value may not be appropriate for all trapping environments of $\mathrm{Ba}$ in the solid RGs, i.e., the violet and red sites. The asymmetric threefold splits observed for
Table 3. Summary of Observed and Corrected Excited State Lifetimes for Each Site of Isolation in All Three RG Systems $^{a}$

\begin{tabular}{cccccccc} 
& & & & \multicolumn{4}{c}{$\tau_{\text {cor }}(\mathrm{ns})$} \\
\cline { 5 - 8 } $\mathrm{RG}$ & site & $\tau_{\text {obs }}(\mathrm{ns})$ & $\tau_{\text {rad }}(\mathrm{ns})$ & $s=0$ & $s=1 / 3$ & $s=1 / 2$ & $s=1$ \\
$\mathrm{Ar}$ & violet & 4.5 & $\sqrt{ }$ & 5.9 & 9.2 & 11.2 & 18.0 \\
& blue & $3.9^{b}$ & $\sqrt{ }$ & 5.1 & 8.0 & 9.7 & 15.6 \\
& green & 4.3 & $\sqrt{ }$ & 5.7 & 8.8 & 10.7 & 17.2 \\
& red & 4.9 & $\sqrt{ }$ & 6.5 & 10.1 & 12.2 & 19.6 \\
$\mathrm{Kr}$ & violet & 4.2 & $\sqrt{ }$ & 6.0 & 10.9 & 13.8 & 24.9 \\
& blue & 4.4 & $\sqrt{ }$ & 6.3 & 11.4 & 14.5 & 26.1 \\
& green & 4.5 & $\sqrt{ }$ & 6.4 & 11.6 & 14.8 & 26.7 \\
$\mathrm{Xe}$ & violet & 4.3 & $\sqrt{ }$ & 6.4 & 12.7 & 16.6 & 31.6 \\
& blue & 3.3 & $\times$ & 4.9 & 9.7 & 12.7 & 24.2 \\
& green & 4.0 & $\sqrt{ }$ & 6.0 & 11.8 & 15.4 & 29.4
\end{tabular}

${ }^{a}$ The effective field correction was made with eq I. With the use of single exponential fits, the uncertainty in the extracted decay times is estimated to be $\pm 0.2 \mathrm{~ns}$. It arises from a convolution of the $5 \mathrm{~ns}$ pulsed laser excitation and the 2 ns iCCD detection. ${ }^{b}$ The decay time obtained for the blue site in $\mathrm{Ar}$ is only tentatively assigned as the radiative lifetime.

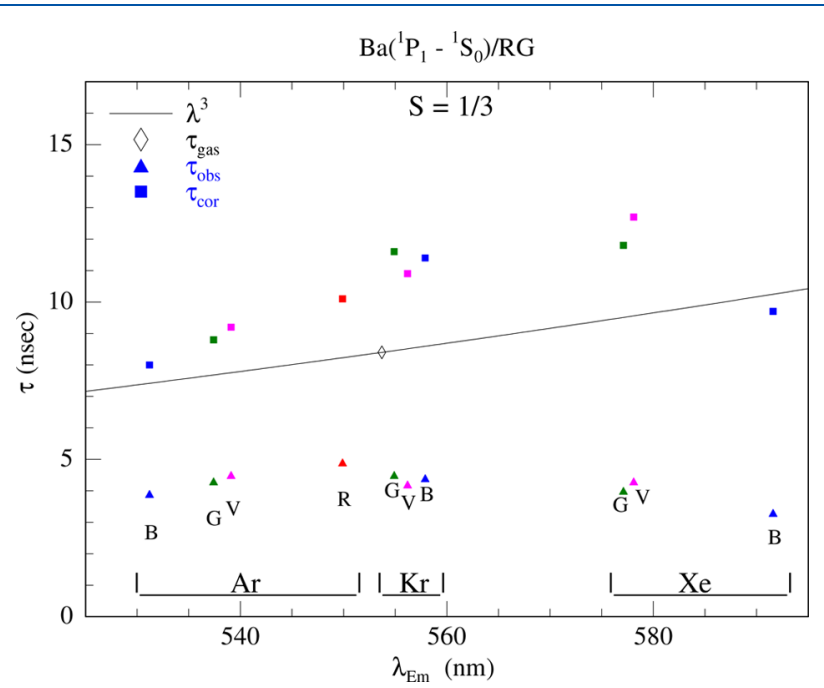

Figure 12. Comparison of emission decay times recorded at $10 \mathrm{~K}$ for the thermally stable Ba: $(6 s 6 \mathrm{p}){ }^{1} \mathrm{P}_{1} \rightarrow{ }^{1} \mathrm{~S}_{0}\left(6 \mathrm{~s}^{2}\right)$ emission features in $\mathrm{Ar}, \mathrm{Kr}$, and $\mathrm{Xe}$ and a $\lambda^{3}$ extrapolation of the gas phase lifetime ${ }^{10}$ for this transition of atomic Ba. With the sole exception of the blue site in $\mathrm{Ba} / \mathrm{Xe}$, all the measured decay times were found to be independent of temperature and accordingly are identified as radiative lifetimes. The uncorrected lifetime values are given by filled triangles, whereas the values corrected for the effective field of the host are denoted by the filled squares.

these sites implies that the RG site accommodating the large $\mathrm{Ba}$ atom is anisotropic. As a test, a number of values for $s$ were used to compute the effective field correction based on a variety of cavity shapes, as described by Shibuya. ${ }^{15}$ For instance, transitions whose moments are considered to be in the longitudinal or transverse direction of a long cylindrical cavity yield values of $s=0$ and $1 / 2$, respectively.

Inspection of the corrected lifetime values provided in Table 3 shows that the values closest to the gas phase $\lambda^{3}$ extrapolation (Figure 12) are those computed with the parameter $s=1 / 3$. However, even these show a deviation and are all, with the exception of the blue site in $\mathrm{Ba} / \mathrm{Xe}$, systematically longer than the gas phase extrapolation. It 
should be noted that the radiative lifetime of this site in xenon was not identified even at $9.8 \mathrm{~K}$, the lowest temperature attainable with the cryostat used, so the recorded value does not provide a valid comparison with the other sites. This model, using just the index of refraction of the solid, is probably too simplistic to take into account the complicated interactions that occur for an excited $\mathrm{Ba}$ atom at a given $\mathrm{RG}$ site and the effects such an interaction has on the oscillator strength of the transition involved. Nonetheless, the measured nanosecond radiative decay times allows confident assignment of the observed $\mathrm{Ba} / \mathrm{RG}$ emission bands to resonance fluorescence of the $(6 s 6 \mathrm{p}){ }^{1} \mathrm{P}_{1} \rightarrow{ }^{1} \mathrm{~S}_{0}\left(6 \mathrm{~s}^{2}\right)$ transition of atomic Ba.

III.h. Site Attributions. The excitation profiles recorded for three $\mathrm{Ba} / \mathrm{RG}$ matrix systems display the richest variety of structures for any matrix-isolated metal atom studied to date. The structures range from the classic Jahn-Teller threefold split bands-best exhibited by the blue site-to asymmetric threefold $(2+1)$ patterns on the violet site, to the apparently structureless bands presented by the green site. The violet, $2+$ 1 bands are observed for all three hosts examined in the present study and, to the best of our knowledge, are unique to the $\mathrm{Ba} / \mathrm{RG}$ matrices. However, from the linear dependence observed in the matrix shift vs matrix polarizability plots, each of these colored sites corresponds to a particular site in the three hosts.

Moreover, on the basis of the results obtained from recent molecular dynamics calculations done on the $\mathrm{Ba} / \mathrm{Xe}$ system, the nature of the sites producing the three main spectral features-violet, blue, and green-can be attributed to particular site types. In the $\mathrm{Ba} / \mathrm{Xe}$ study, strong evidence emerged from comparisons of the recorded excitation spectra with spectral simulations that the blue site was due to a symmetrical tetravacancy (TV) site and the green site was due to a hexavacancy (HV) site in the crystalline fcc lattice. These $\mathrm{Ba} / \mathrm{Xe}$ predictions are consistent with the pronounced threefold splitting observed on the blue sites in $\mathrm{Kr}$ and $\mathrm{Xe}$ and also with the three component fits done on the green site for all three solids. Thus, in the cuboctahedral fcc lattice, the TV and HV multivacancies result in high symmetry $O_{h}$ and $T_{d}$ sites producing the characteristic Jahn-Teller splitting on the isolated guest metal atom. More uniquely, the molecular dynamics calculations indicated that the trapping site responsible for the $2+1$ pattern of the $\mathrm{Ba} / \mathrm{Xe}$ violet site corresponds to a Ba atom isolated at a grain boundary (GB). In solid xenon this site consists of a five-atom vacancy with $D_{3 h}$ symmetry, located at the boundary of two fcc slabs. From the similar patterns observed for the violet site in $\mathrm{Kr}$ and $\mathrm{Ar}$ and the linear matrix shift vs polarizability plots, it is highly likely that this site is also present in both of these solids.

Attributions of the thermally stable red sites that exist in $\mathrm{Ba} /$ $\mathrm{Ar}$ and the partially stable red sites in $\mathrm{Ba} / \mathrm{Kr}$ are less clear. While a large variety of multivacancy sites can exist at grain boundaries, Monte Carlo simulations conducted on $\mathrm{Na}$ atoms in solid $\mathrm{Ar}^{18}$ showed that matrix sites possessing a strong permanent axial asymmetry, such as a cylindrical vacancy, yielded a widely split "doublet plus singlet" absorption line shape. The $\mathrm{R} 1$ trapping sites in Ar and $\mathrm{Kr}$ which give rise to the $2+1$ splitting of the excited $\mathrm{P}$ state of $\mathrm{Ba}$ must be characterized by a lower symmetry since the doublet component exhibits a small splitting. Recent molecular dynamics simulations, conducted by Gervais et al. ${ }^{19}$ of the alkali metal atoms embedded in Ar, may offer insight into such vacancies. The study revealed that the "red" absorption bands of large atoms, such as potassium, arise from the occupation of a number of distinct grain boundary (GB) sites in Ar. Indeed these sites were shown to be more favorable than larger vacancies in the bulk solid. The authors report that more compact GB cavities with reduced dimensionality systematically exhibit one or more peaks shifted toward higher energy. It is plausible that such a vacancy could reproduce the excitation/ absorption patterns intrinsic to the occupation of a $\mathrm{Ba}$ atom in the R1 sites. On the other hand, larger GB cavities can produce broad unstructured bands and triplet structures (not arising from a JT effect) - which could be correlated to the R2 matrix site.

However, the GB sites are known ${ }^{19}$ to be quite sensitive to matrix annealing. This behavior closely matches the relatively low annealing threshold observed for the red (R1 and R2) site features in $\mathrm{Ar}$ and $\mathrm{Kr}$ but is in conflict with the high stability of the violet sites in all three hosts. From the existing theoretical work done on $\mathrm{Ba} / \mathrm{Xe}$, it is proposed that the violet sites of isolation occur at a $\mathrm{GB}$ vacancy that involves the minimum loss of structure within the bulk RG solid. For $\mathrm{Ba} / \mathrm{Xe}$, a compact five-atom vacancy which maintains threefold symmetry of the fcc lattice was identified as the origin of the violet band. Based on the observed matrix shifts, the red excitation features (R1) in $\mathrm{Ar}$ and $\mathrm{Kr}$ should correspond to the isolation of $\mathrm{Ba}$ in larger GB cavities. The exact nature of the red sites requires input from $\mathrm{MD}$ calculations and spectral simulations. It is hoped future work should be revealing of this dominant site in $\mathrm{Ba} / \mathrm{Ar}$.

\section{CONCLUSION}

The 2D-EE spectra recorded in this study have allowed a comprehensive yet detailed examination to be conducted of the sites occupied by $\mathrm{Ba}$ atoms in the three solid rare gases $\mathrm{Ar}$, $\mathrm{Kr}$, and Xe. The excitation scans extracted from the 2D-EE spectra reveal that the site occupancy of barium in the RG solids is much more complex than the absorption spectroscopy would suggest. Three thermally stable sites-labeled blue, green, and violet-have been identified in all three solids. The amounts of these sites vary from solid to solid-with the blue site dominating in $\mathrm{Xe}$ while it is the green in $\mathrm{Ar}$. In $\mathrm{Ba} / \mathrm{Kr}$ the three sites are present in roughly similar quantities.

The temperature dependence of the excited state lifetimes was found to depend primarily on the RG host, but also on the site of isolation. For $\mathrm{Ba} / \mathrm{Xe}$, a shortening of the site-specific emission lifetimes at elevated temperatures was observed ${ }^{1}$ and tentatively attributed a ${ }^{1} \mathrm{P}_{1} \rightarrow{ }^{3} \mathrm{P}_{2}$ intersystem crossing. This effect was most pronounced for the blue site of isolation, where the radiative lifetime was not identified, even at $10 \mathrm{~K}$. In contrast, changes in the decay profiles were not observed in Ar and $\mathrm{Kr}$, over the temperature ranges assessed. The efficiency of intersystem crossing in solid $\mathrm{Xe}$ is proposed to arise from the external heavy atom effect. ${ }^{8}$

\section{AUTHOR INFORMATION}

\section{Corresponding Author}

*E-mail: john.mccaffrey@mu.ie.

\section{ORCID}

John G. McCaffrey: 0000-0002-6770-7743

\section{Notes}

The authors declare no competing financial interest. 


\section{ACKNOWLEDGMENTS}

This research was in part funded by the John \& Pat Hume award at Maynooth University which provided Barry Davis with a Ph.D. studentship.

\section{REFERENCES}

(1) Davis, B. M.; Gervais, B.; McCaffrey, J. G. An investigation of the sites occupied by atomic barium in solid xenon-A 2D-EE luminescence spectroscopy and molecular dynamics study. J. Chem. Phys. 2018, 148, 124308

(2) Balling, L.; Wright, J. Absorption and emission spectra of matrixisolated Ba atoms. J. Chem. Phys. 1985, 83, 2614-2615.

(3) Mong, B.; et al. Spectroscopy of $\mathrm{Ba}$ and $\mathrm{Ba}^{+}$deposits in solid xenon for barium tagging in nEXO. Phys. Rev. A: At., Mol., Opt. Phys. 2015, 91, 022505.

(4) Davis, B. M.; McCaffrey, J. G. Absorption spectroscopy of heavy alkaline earth metals $\mathrm{Ba}$ and $\mathrm{Sr}$ in rare gas matrices-CCSD (T) calculations and atomic site occupancies. J. Chem. Phys. 2016, 144, 044308.

(5) Collier, M. A.; McCaffrey, J. G. The absorption and excitation spectroscopy of matrix-isolated atomic manganese: sites of isolation in the solid rare gases. J. Chem. Phys. 2005, 122, 054503.

(6) Byrne, O.; McCaffrey, J. G. Site-selected luminescence of atomic europium in the solid rare gases. J. Chem. Phys. 2011, 135, 024507.

(7) Laursen, S. L.; Cartland, H. E. Multiplicity dependence of matrix-induced frequency shifts for atomic transitions of the group 12 metals in rare gas solids. J. Chem. Phys. 1991, 95, 4751-4755.

(8) Crepin-Gilbert, C.; Tramer, A. Photophysics of metal atoms in rare-gas complexes, clusters and matrices. Int. Rev. Phys. Chem. 1999, $18,485-556$.

(9) Ryan, M.; et al. Investigations of the optical spectroscopy of atomic sodium isolated in solid argon and krypton: experiments and simulations. J. Phys. Chem. A 2010, 114, 3011-3024.

(10) Kramida, A.; Ralchenko, Y.; Reader, J. NIST ASD Team NIST atomic spectra database, version 5.3; National Institute of Standards and Technology: Gaithersburg, MD. Available at http://physics.nist. gov/asd. July 20, 2017.

(11) Lebedev, V.; Moroshkin, P.; Weis, A. Spectroscopy of barium atoms in liquid and solid helium matrices. Phys. Rev. A: At., Mol., Opt. Phys. 2011, 84, 022502.

(12) Davis, B. M. Luminescence spectroscopy of strontium and barium atoms isolated in low temperature solids - an experimental and theoretical study Ph.D. Dissertation, Maynooth University, 2016.

(13) Visticot, J.; et al. Experiment versus molecular dynamics simulation: Spectroscopy of $\mathrm{Ba}-(\mathrm{Ar}) \mathrm{n}$ clusters. J. Chem. Phys. 1994, $100,158-164$.

(14) Bracken, V. A.; Gürtler, P.; McCaffrey, J. G. Luminescence spectroscopy of atomic zinc in rare-gas solids. I. J. Chem. Phys. 1997, 107, 5290-5299.

(15) Shibuya, T. i. A dielectric model for the solvent effect on the intensity of light absorption. J. Chem. Phys. 1983, 78, 5175-5182.

(16) Fulton, R. L. Optical properties of a guest molecule in a discrete lattice. J. Chem. Phys. 1974, 61, 4141-4155.

(17) The refractive indices of $\mathrm{Ar}, \mathrm{Kr}$, and $\mathrm{Xe}$ are 1.29, 1.28, and 1.49 recorded at $60 \mathrm{~K}$ and $\lambda=488 \mathrm{~nm}$. See: Jodl, H. J. Solid State Aspects of Matrices. In Chemistry and Physics of Matrix-Isolated Species; Andrews, L., Moskovitz, M., Eds.; North-Holland: 1989.

(18) Boatz, J. A.; Fajardo, M. E. Monte Carlo simulations of the structures and optical absorption spectra of $\mathrm{Na}$ atoms in Ar clusters, surfaces, and solids. J. Chem. Phys. 1994, 101, 3472.

(19) Jacquet, E.; et al. Spectroscopic properties of alkali atoms embedded in Ar matrix. J. Chem. Phys. 2011, 135, 174503. 